\title{
Altered Negative BOLD Responses in the Default-Mode Network during Emotion Processing in Depressed Subjects
}

\author{
Simone Grimm', Peter Boesiger², Johannes Beck', Daniel Schuepbach', Felix Bermpohl ${ }^{3}$, Martin Walter $^{4}$, \\ Jutta Ernst ${ }^{4}$, Daniel Hell', Heinz Boeker' and Georg Northoff $*, 1,4$ \\ 'Department of Psychiatry, University of Zurich, Zurich, Switzerland; 'Division of Biophysics, Institute of Biomedical Engineering, ETH and \\ University of Zurich, Zurich, Switzerland; ${ }^{3}$ Department of Psychiatry, Charité, Humboldt University of Berlin, Berlin, Germany; ${ }^{4}$ Department of \\ Psychiatry, University of Magdeburg, Magdeburg, Germany
}

\begin{abstract}
Studies using functional magnetic resonance imaging ( $\mathrm{fMRI}$ ) show predominant negative blood oxygenation level-dependent (BOLD) responses (NBRs) in regions of the default-mode network such as the pregenual anterior cingulate cortex, the ventromedial prefrontal cortex, and the posterior cingulate cortex. Patients with major depressive disorder (MDD) show emotional-cognitive disturbances, which have been associated with alterations within the default-mode network. However, it remains unclear whether these default-mode network alterations are related to abnormalities in NBRs. We therefore investigated neural activity in the default-mode network during different emotional tasks in patients with MDD in an event-related $\mathrm{AMRI}$ design. MDD patients showed significantly reduced NBRs in several regions of the default-mode network. Decreased NBRs in MDD patients correlated with depression severity and feelings of hopelessness. In sum, our findings demonstrate that default-mode network NBRs are reduced in MDD and modulate these patients' abnormally negative emotions.

Neuropsychopharmacology (2009) 34, 932-943; doi: I0.1038/npp.2008.8I; published online 4 June 2008
\end{abstract}

Keywords: default-mode network; negative BOLD responses; major depressive disorder; event-related fMRI; emotional processing

\section{INTRODUCTION}

The mesial cortex or the so-called cortical midline structures (CMS) have increasingly become a focus of attention in neuroimaging. The CMS, which include the ventromedial prefrontal cortex (VMPFC), the perigenual anterior cingulate cortex (PACC), and the posterior cingulate cortex (PCC) are characterized by negative blood oxygenation level-dependent (BOLD) responses (NBRs) rather than positive BOLD responses (PBRs) during various emotional-cognitive tasks (Gusnard et al, 2001; McKiernan et al, 2003; Northoff et al, 2004; Raichle and Gusnard, 2005; Fransson, 2005; Grimm et al, 2006). Together with other cortical regions (dorsomedial prefrontal cortex, lateral parietal cortex, and superior temporal gyrus) these areas are considered part of the default-mode network (Raichle et al, 2001; Raichle and Gusnard, 2005) which is characterized by high neuronal resting- state activity and inhibited in tasks that require subjects to attend to external stimuli (Greicius et al, 2004; Fransson, 2005; Fox et al, 2005, 2006).

*Correspondence: Professor G Northoff, Department of Psychiatry, University of Magdeburg, Leipziger Strasse 44, Magdeburg, SacshsenAnhalt 39120, Germany, Tel: + 0049 39| 67|4234, Fax: + 004939 | 67| 5223, E-mail: georg.northoff@medizin.uni-magdeburg.de Received 19 December 2007; revised 21 April 2008; accepted 24 April 2008
Regions that belong to the default-mode network also show alterations in neuronal activity in major depressive disorder (MDD), a disease that is characterized by strong feelings of sadness, guilt, worthlessness, and hopelessness (Raes et al, 2006; Treynor, 2003; Rimes and Watkins, 2005). Imaging studies in MDD demonstrated structural abnormalities as well as increased signal intensities in ventral CMS. Increased activity has mainly been found in the subgenual cingulate, PACC, and VMPFC (Rajkowska, 2002, 2003; Mayberg, 2003; Canli et al, 2004; Lawrence et al, 2004; Surguladze et al, 2005; Beauregard et al, 2006). Increased activity in the anterior cingulate was shown to be associated with severity of depressive symptoms, neuropsychological deficits, and response to antidepressants (Dunn et al, 2002; Kimbrell et al, 2002; Kennedy et al, 2001; Davidson et al, 2003). A recent resting-state functional magnetic resonance imaging (fMRI) study by Greicius et al (2007) investigated the default-mode network in MDD patients and demonstrated a significantly greater subgenual cingulate and thalamic functional connectivity, which was disproportionately driven by activity in the subgenual cingulate. Depression refractoriness, as measured by the length of the current episode, was positively correlated with functional connectivity in the subgenual cingulate. Though these investigations suggest functional alterations in the default-mode network, altered NBRs during emotional processing remain to be investigated in MDD. We aimed at investigating NBRs 
in ventral CMS regions in MDD. As resting-state abnormalities of the default-mode network in MDD as well as increased signal intensities during different emotionalcognitive tasks have been demonstrated by previous studies (Mayberg, 2003; Surguladze et al, 2005, Beauregard et al, 2006; Greicius et al, 2007), we hypothesized abnormal NBRs in MDD patients. To induce default-mode NBRs, we relied on a previously successfully established paradigm that probed two external emotional stimulations (emotional perception (EP) and emotional judgment (EJ)) and an internal one (emotional expectancy) (Grimm et al, 2006; Bermpohl et al, 2006; Heinzel et al, 2005). We hypothesized that MDD patients show reduced NBRs in the default-mode network during the external emotional tasks, whereas no abnormal signal changes were expected in the internal condition. As previous studies implicated default-mode network NBRs in negative emotion processing and its interaction with the self (Heinzel et al, 2005; Grimm et al, 2006; Lewis et al, 2006; Phan et al, 2004; Moran et al, 2006), we hypothesized that reduced NBRs are related to depressive symptoms (hopelessness and depressive cognitions) and depression refractoriness (duration of the current episode and number of previous episodes).

\section{MATERIALS AND METHODS}

\section{Subjects}

Subjects with an acute MDD episode were recruited from the in-patient Department of Psychiatry at the University of Zurich. Eligibility screening procedures included the 21-item Hamilton Depression Rating Scale (HDRS) (Hamilton, 1960), the 21-item Beck Depression Inventory (BDI) (Beck et al, 1961), the 20-item Beck Hopelessness Scale (BHS), (Beck et al, 1974) and clinical laboratory tests. Diagnoses of depression were made by the participants' treating psychiatrists. Family history was not obtained and therefore not used in the inclusion/exclusion criteria. Inclusion criteria were a score of at least 24 on the HDRS and the BDI. These criteria were designed to ensure inclusion of participants with similar levels of endogenous-type symptoms. Ratings were conducted by psychiatrists or research assistants who had demonstrated adequate inter-rater reliability. Exclusion criteria were major medical illnesses, histories of seizures, head trauma with loss of consciousness, abnormal clinical laboratory tests, and pregnancy. Additionally, patients who were actively suicidal, met criteria for any psychiatric disorder other than MDD, had a history of substance abuse or electroconvulsive therapy in the previous 6 months, or had a history of substance dependence were excluded from the study. Patients had been free of psychotropic medication for at least 1 week prior to scanning. Healthy subjects without any psychiatric, neurologic, or medical illness were self-referred from online study advertisements. Control subjects were paid 30 Swiss Francs/h. The study was approved by the University of Zurichs' Institutional Review Board, and all subjects gave written informed consent before screening. All subjects were right-handed as assessed with the Edinburgh Handedness Inventory (Oldfield, 1971). After applying the exclusion criteria above, fMRI scans from 20 depressed subjects and 30 control subjects were processed. Of these scans, two could not be included in the analysis owing to structural abnormalities in the 3D T1-weighted anatomical scan (one depressed subject and one control). This resulted in usable fMRI data on 19 subjects with depression and 29 control subjects.

\section{Pictorial Stimuli}

Subjects viewed full-color pictures selected from the International Affective Picture System (IAPS) (Lang et al, 1999 ) with positive (IAPS norm ratings: $7.32 \pm 2.06$ ) and negative (IAPS norm ratings: $2.24 \pm 2.67$ ) valence. Positive pictures included stimuli such as smiling babies, flowers, and landscapes. Negative pictures included stimuli such as burn victims, snakes, and weapons pointed at others as well as at the viewer. The picture sets were counterbalanced across all subjects as well as within each subject according to the two categories of valence as well as according to dominance, intensity, human faces, and human figures. All pictures were centered on a black background. The pictures were generated by an in-house software (SCOPE V2.5.4 Display Program; Max R Duersteler, University Hospital Zurich, Switzerland) and rear projected onto a projection screen positioned at the head end of the MRI scanner bore. Subjects viewed the screen through a mirror mounted on the head coil and responded by pushing a fiber-optic light-sensitive keypress.

\section{Experimental Design}

The fMRI design was 'event related' with emotional stimulation alternating with a fixation control condition and an expectancy condition (Figure 1). The IAPS pictures serving for emotional stimulation were presented for $4 \mathrm{~s}$. In half of the presented pictures, subjects had to judge them as to whether they were positive or negative in content ('EJ'); this was indicated by the letters ' $\mathrm{P} / \mathrm{N}$ ' in one corner of the picture. Subjects were to press the keypress button under the index finger of their left hand, if the picture was judged as negative and to press the button under the index finger of their right hand, if the picture was judged as positive. In the other half of the presented pictures, subjects had to passively view the picture ('EP'); this was indicated by the letters 'A/A' in one corner of the picture. Here, subjects were instructed to arbitrarily press a button with their left or right index finger without making any judgment to control for movement effects. Responses and response times were recorded by the SCOPE program. Response times were calculated from stimulus onset to the keypress response. We did not include a non-emotional control condition, eg neutral IAPS pictures for two reasons. First, our focus was on the comparison of viewing and judgment of emotional pictures with baseline, respectively, rather than on the comparison of emotional and neutral pictures. Second, neutral pictures show lower intensity ratings than emotional pictures; differences in intensity between emotional and neutral pictures might thus have interfered with a valid comparison between viewing/judgment and baseline. In $50 \%$ of the trials, the pictures were preceded by a resting period (presentation of a fixation cross) and therefore viewed and judged in an unexpected condition ('unexpEP' and 'unexpEJ'). In contrast, the other $50 \%$ of the pictures were preceded by an expectancy period of $8-11.5 \mathrm{~s}(8.0,8.5$, 


\begin{tabular}{|c|c|c|}
\hline $\begin{array}{c}\text { Expectancy:Emotion } \\
\text { Judgment: 8-11.5 s }\end{array}$ & $\begin{array}{c}\text { Emotion Judgment: } \\
4 \mathrm{~s}\end{array}$ & $\begin{array}{c}\text { Fixation cross: } \\
6-8 \mathrm{~s}\end{array}$ \\
\hline & $\begin{array}{c}\text { Emotion Judgment: } \\
4 \mathrm{~s}\end{array}$ & $\begin{array}{c}\text { Fixation cross: } \\
6-8 \mathrm{~s}\end{array}$ \\
& & \\
& Emotion Perception: & $\begin{array}{c}\text { Fixation cross: } \\
6-8 \mathrm{~s}\end{array}$ \\
\hline $\begin{array}{l}\text { Expectancy: Emotion } \\
\text { Perception: 8-11.5 s }\end{array}$ & $4 \mathrm{~s}$ & $\begin{array}{c}\text { Fixation cross: } \\
6-8 \mathrm{~s}\end{array}$ \\
\hline
\end{tabular}

Figure I Schematic representation of the fMRI paradigm for emotion perception (EP) and emotion judgment (EJ) of IAPS pictures. Pictures in both conditions (EP and EJ) were presented either with or without a preceding expectancy period of $8-11.5 \mathrm{~s}$. After the picture presentation of $4 \mathrm{~s}$, a fixation cross, indicating the resting period, was presented for 6-8s.

9.0, 9.5, 10.0, 10.5, 11.0, and 11.5 s) ('expEP' and 'expEJ'), indicating the type of task associated with the picture subsequently presented. The purpose of this preceding expectancy period was to include an internally oriented condition that mostly relied on internal stimulation with mental stimuli originating in the subject itself. The expectancy period was indicated by presentation of a white fixation cross on a dark background and a letter in one of the corners of the picture. ' $J$ ' indicated expectancy of a subsequent judgment task, whereas ' $E$ ' was associated with expectancy of subsequent picture perception. To prevent fear or appetitive conditioning (Bechara et al, 1995), the emotional expectancy cue did not provide information as to whether a positive or negative emotional picture would follow. After the presentation of each picture, a resting period followed, where a fixation cross was presented for $6-8 \mathrm{~s}(6.0,6.5,7.0,7.5$, and $8.0 \mathrm{~s})$. This allowed the subjects to recover from emotional stimulation and, in addition, served as a baseline condition to distinguish between positive and negative BOLD responses (Stark and Squire, 2001). Subjects were instructed to fixate on the crosshair when it was present. The duration of the resting period was randomly varied accounting for variable stimulus onset asynchrony. A total of 158 trials were presented in six runs; 79 trials were presented for EP (28 trials for unexpEP and 51 trials for expEP) and 79 trials for EJ (28 trials for unexpEJ and 51 trials for expEJ). The different task conditions were pseudorandomized within and across the six runs and their order counterbalanced across all subjects. Prior to the experimental session, the subjects were familiarized with the paradigm by completing a test run of 10 trials. Immediately after the fMRI session a selection of pictures were presented for a second time. Each of the 151 pictures (including 60 new ones for distraction) was followed by a task period, which consisted of an emotional intensity rating, valence rating, and recognition test. All three responses were given using a scale ranging from 1 to 9 . Valence assessment ranged on a continuum from 'very negative' (1) to 'very positive' (9). Emotional intensity rating scores ranged on a continuum from 'low' (1) to 'high' (9). For recognition of emotional pictures, choices ranged on a continuum from 'definitely not recognized' (1) to 'definitely recognized' (9). The 60 new emotional pictures were matched in valence, intensity, and dominance with those presented in fMRI. It is possible that emotional responses may attenuate when pictures are seen for a second time.
However, this habituation effect applies equally to all picture conditions and should not affect the differences between conditions (Anderson et al, 2003). The mean average of the three ratings was calculated for each subject. Analysis of postscanning ratings was conducted separately for positive and negative pictures. Postscanning ratings were conducted with 28 healthy controls and 13 depressed subjects, as some of the subjects were too exhausted after the fMRI scan to continue the investigation.

Functional imaging. Measurements were performed on a Philips Intera 3T whole-body MR unit equipped with an eight-channel Philips SENSE head coil. Functional time series were acquired with a sensitivity encoded (Pruessmann et al, 1999) single-shot echo-planar sequence (SENSE-sshEPI). SENSE makes use of receiver coil arrays for image acquisition, which allow to reconstruct the full-field-of-view (FOV) images from undersampled data by using information from spatially varying coil sensitivity profiles. In turn, the undersampling leads to shorter echo train length and faster $k$-space coverage. SENSE compared to conventional EPI has been shown to considerably reduce susceptibility related image distortion and signal dropout (Schmidt et al, 2005). The following acquisition parameters were used in the fMRI protocol: TE (echo time) $=35 \mathrm{~ms}$, $\mathrm{FOV}=22 \mathrm{~cm}$, acquisition matrix $=80 \times 80$, interpolated to $128 \times 128$, voxel size $=2.75 \times 2.75 \times 4 \mathrm{~mm}^{3}$, and SENSE acceleration factor $R=2.0$. Using a mid-saggital scout image, 32 contiguous axial slices were placed along the anterior-posterior commissure plane covering the entire brain with a $\mathrm{TR}=3000 \mathrm{~ms} \quad\left(\theta=82^{\circ}\right)$. The first three acquisitions were discarded due to T1 saturation effects.

\section{Statistical Analysis}

Behavioral data. Reaction times and judgments (positive/ negative rating) were analyzed in a multivariate ANOVA with the factors group (healthy subjects/MDD patients), valence (positive/negative pictures), task (EJ/EP), and expectancy (with/without expectancy period). Postscanning ratings of valence, intensity, and recognition were analyzed in a group $\times$ valence ANOVA.

fMRI data. Data were analyzed using the SPM2 software (www.fil.ion.ucl.ac.uk/spm/). Functional data were corrected for differences in slice acquisition time, realigned to the 
first volume, corrected for motion artifacts, mean-adjusted by proportional scaling, normalized into standard stereotactic space (template provided by the Montreal Neurological Institute), and spatially smoothed using a $8 \mathrm{~mm}$ FWHM Gaussian kernel. The time series were high-pass filtered to eliminate low-frequency components (filter width $128 \mathrm{~s}$ ) and adjusted for systematic differences across trials. Statistical analysis was performed by modeling the different conditions (unexpected/expected EJ (unexpEJ and expEJ), unexpected/expected EP (unexpEP and expEP), expectancy periods preceding judgment/picture perception (exEJ and exEP), and resting period) convolved with a hemodynamic response function as explanatory variables within the context of the general linear model on a voxel-by-voxel basis. Realignment parameters were included as additional regressors in the statistical model. A fixed-effect model at a single-subject level was performed to create images of parameter estimates, which were then entered into a second-level random-effects analysis. To detect betweengroup differences, the contrast images of all subjects of each group (depressed and control subjects) were included in a two-sample $t$-test (threshold $p<0.001$, uncorrected and at least five active voxels). fMRI analysis focused on the comparison of the different emotional conditions with the resting condition to reveal NBR. In the first step, we applied whole-brain analysis to get an overview over all regions showing NBR. Then region of interest (ROI) analyses were performed to further investigate NBRs in these regions and to correlate them with valence and psychopathological parameters. We did not attend to differences between positive and negative emotional stimuli, as the main focus of our study was on the investigation of NBRs as induced by emotional stimulation and not on the distinction between the processing of positive and negative stimuli. No subject had to be excluded due to susceptibility artifacts or significant signal dropout. To exclude the possible influence of unequal sample size between healthy $(n=29)$ and MDD $(n=19)$ subjects, the depressed subjects were compared to an age-matched sample of 19 healthy controls. Results replicated those obtained from the full sample $(n=29)$. Similarly, inclusion of age, gender, psychotropic medication, and length of wash-out period as covariates did not have any influence on the results from group comparisons. To determine whether subjects activated overlapping or dissociable neuronal networks during EJ and EP, the two contrasts of all subjects of each group were entered into a random-effect conjunction analysis to determine areas of activation common to both conditions (Price and Friston, 1997). Clusters of activation were identified with a global height threshold of $p<0.05$ (corrected for family-wise errors (FWE)) and an extent threshold of $k=10$ voxels. Exclusive masking was applied to the conditions of interest, ie EP/EJ respectively, to eliminate voxels activated by the respective condition. The contrast rest $>$ EJ was exclusively masked with the contrast rest $>E P$ (and vice versa: rest $>E P$ was exclusively masked with rest $>E J)$. The significance level of the masks was set at $p<0.05$ uncorrected. Clusters of activation were identified with a global height threshold of $p<0.05$ (corrected for FWE) and an extent threshold of $k=10$ voxels. FWE correction was chosen because it provides a more conservative approach than the FDR correction that uses an adaptive threshold based upon both the number of tests and the distribution of the uncorrected $p$-values of those tests (Logan and Rowe, 2004). For the ROI analyses of peak voxels, coordinates that were obtained in contrasts of the between-group analyses (Table 1) were selected. Regions of interest were functionally defined by centering spheres on the respective peak voxels with a radius of $10 \mathrm{~mm}$. Analyses were carried out for the VMPFC $(12,52$, and -2$)$, pregenual anterior cingulate cortex (PACC) $(10,46$, and -8$)$ and the dorsal PCC $(8,-10$, and 46). For the ROI analyses, effect sizes (\% signal change) for the different conditions were extracted for each subject separately using Marsbar (http://marsbar.sourceforge.net/). The Marsbar toolbox uses the statistics machinery provided by SPM. For each event, \% signal changes were calculated relative to the mean signal intensity of this ROI across the whole experiment. Time course analyses were performed applying a finite impulse response model that does not make an assumption on the resulting signal changes after stimulus presentation (Ollinger et al, 2001). Parameter estimates of the peri-stimulus time histogram were calculated for eight time bins of 1TR $(=3 \mathrm{~s})$ length for each regressor of our design matrix. To detect the association of signal changes in response to emotional stimulation with valence, individual contrast images of the contrast rest $>$ all pictures (comprised of EP and EJ) were correlated with scores from individual postscanning ratings of emotional valence (Grimm et al, 2006; Anderson et al, 2003). Correlation was assessed using SPM2 simple regression analysis $(p<0.005$ uncorrected, $k>10)$. To test the a priori hypothesis of a dysfunction in the CMS, a ROI approach focusing on PACC, VMPFC, and PCC was pursued. The effect sizes in these regions were extracted using Marsbar (see above) and then correlated with the individual scores using Pearson correlation analysis. All correlation analyses were calculated separately for healthy and MDD subjects. To analyze the difference in $r$ values, correlation coefficients were compared using Fischer's $z$-transformation. To detect the association of signal changes in response to emotional stimulation with psychopathological parameters and parameters of depression refractoriness, the correlation between duration of the current episode, number of previous episodes, patients' scores on the HDRS, BDI, BHS, and signal changes in PACC $(10,46$, and -8), VMPFC $(12,52$, and -2$)$ and PCC $(8,-10$, and 46) was analyzed in a post hoc, ROI analysis using Marsbar (see above). We also assessed the association with the different psychopathological components of the HDRS (five-factor solution; Milak et al, 2005). MDD patients' individual scores were correlated with signal changes during rest $>$ all pictures (comprised of EP and EJ) using Pearson correlation analysis.

\section{RESULTS}

\section{Subjects}

The control group had a mean age of 35.2 years with 21 women and 8 men. The depressed group had a mean age of 40.0 years with 11 women and 8 men. Groups did not differ significantly in age ( $t$-test $p=0.07)$ or gender distribution $\left(\chi^{2} p=0.35\right)$. The mean HDRS score was 33.1 (SD 7.1), the mean BDI score was 29.9 (SD 4.9) and the mean BHS score was 33.63 (SD 3.6) in the depressed group. The mean 
Table I Differences between Healthy and Depressed subjects in Emotion Conditions (EP, EJ, ExEP, and ExEJ) when Compared to the Resting Condition

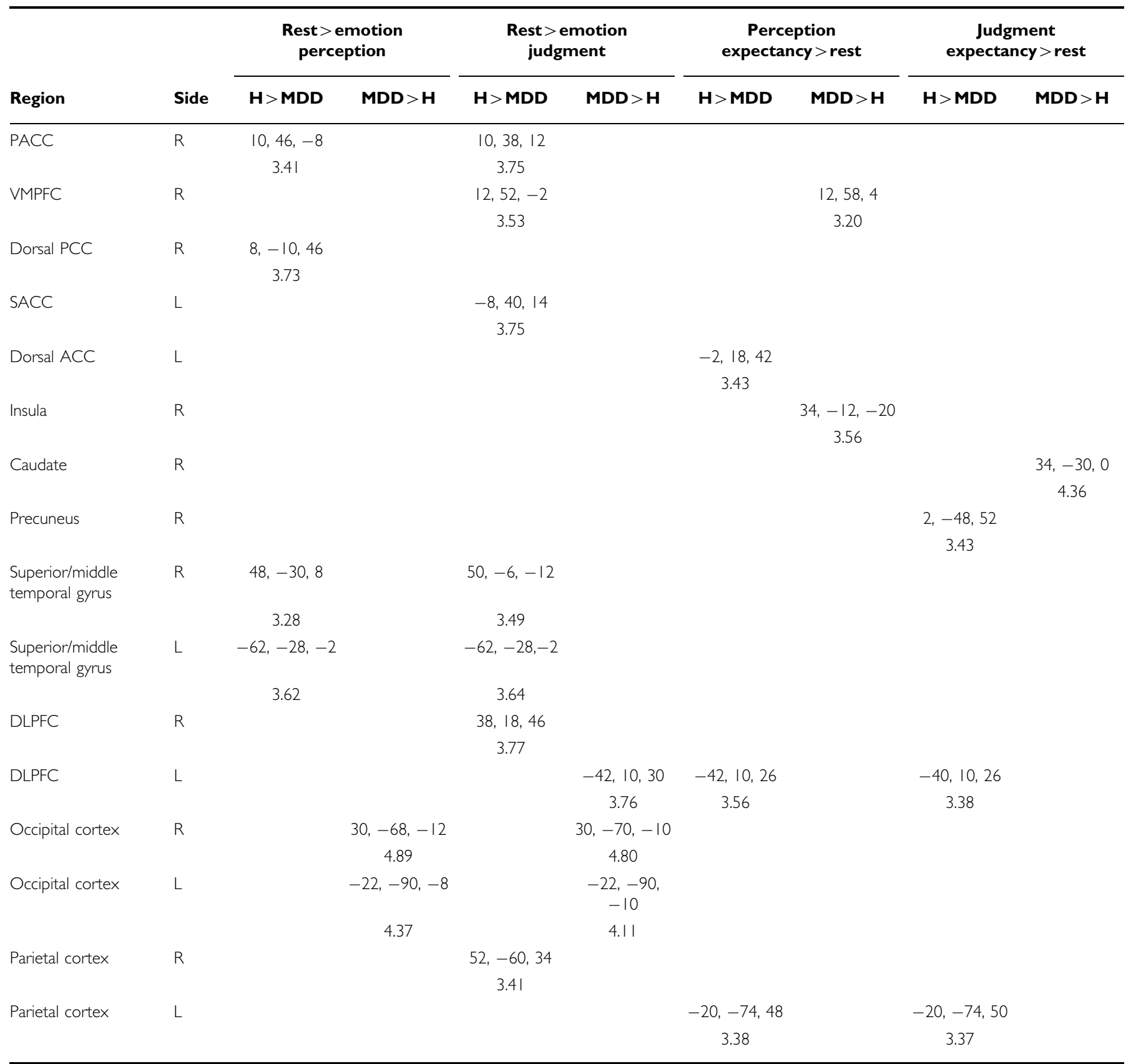

Abbreviations: ACC, anterior cingulate cortex; DLPFC, dorsolateral prefrontal cortex; MDD, MDD patients; PACC, pregenual anterior cingulate cortex;

PCC, posterior cingulate cortex; SACC, supragenual anterior cingulate cortex; VMPFC, ventromedial prefrontal cortex.

The global height threshold for between-group comparisons (healthy versus MDD) was set to $p<0.00 \mathrm{I}$ uncorrected, the extent threshold to $k=5$ voxels for all contrasts. The values in the table represent maximum $z$ values with peak voxel coordinates in the MNI stereotactic space.

HDRS score was 3.6 (SD 1.5), the mean BDI score was 3.8 (SD 3.0) and the mean BHS score was 4.5 (SD 3.7) in the control group. With regard to exposure to psychotropic medications, control subjects and depressed subjects were not taking any medications at the time of investigation. In total, 3 of the 19 depressed subjects had never taken antidepressants. Patients had been free of psychotropic medication for at least 1 week prior to scanning (9.1 days, SD 7.9 days). The mean duration of the current episode was
15.8 weeks (SD 16.2), the number of previous depressive episodes was 1.8 (SD 2.2).

\section{Behavioral Data}

Intrascanner ratings (reaction times and judgments). There was a significant effect of group $(\mathrm{F}(1)=145.37$, $p<0.001)$ and task $(\mathrm{F}(1)=6.68, p<0.001)$, but not of valence $(\mathrm{F}(1)=0.93, p=0.33)$ and expectancy $(\mathrm{F}(1)=2.44, p=0.11)$ 
on reaction times. Post hoc $t$-tests demonstrated faster reaction times in healthy subjects in EJ $(t=-8.75, p<0.001)$ as well as in EP $(t=-7.94, p<0.001)$. Concerning the judgments there was a significant group effect $(\mathrm{F}(1)=74.66$, $p<0.001)$ and valence effect $(\mathrm{F}(1)=3634.48, p<0.001)$, but no effect of expectancy $(\mathrm{F}(1)=0.59, p=0.44)$. Differences in judgment between groups mainly concerned the positive pictures, which were rated significantly less positive by the MDD patients $(t=-2.45, p=0.01)$. The results are indicative of a consistent psychomotor impairment as well as a negative bias in patients with MDD.

During EP, where subjects were asked to respond with either hand, answers were symmetrical with respect to positive and negative stimuli. Comparison between groups showed significantly more symmetrical responses for negative stimuli in healthy subjects $\left(\chi^{2} p<0.001\right)$ and for positive stimuli in MDD patients $\left(\chi^{2} p<0.001\right)$.

Postscanning ratings. There was a significant effect of both participant group (healthy and MDD) and picture valence (positive and negative) on ratings of intensity $\mathrm{F}(1)=82.13$, $p<0.001)$, recognition $(\mathrm{F}(1)=14.61, p<0.001)$, and valence $\mathrm{F}(1)=20.32, p<0.001)$. Positive $(t=3.69, p<0.001)$ and negative pictures $(t=2.22, p=0.02)$ were rated significantly more negative by the depressive patients. MDD patients rated positive $(t=5.68, p<0.001)$ and negative pictures $(t=6.14, p<0.001)$ less intense and recognized significantly less negative pictures $(t=3.84, p<0.001)$ than healthy subjects. There were no differences between groups for recognition of positive pictures.

\section{fMRI Data}

Depressed subjects vs controls. Locations of responses are summarized in Table 1. For the contrast rest $>E P$, significantly decreased NBRs in the PACC and the dorsal part of the PCC were found in depressed compared to healthy subjects. The contrast rest $>$ EJ also revealed significantly decreased NBRs in the PACC, which extends into the VMPFC and the SACC in depressed compared to healthy subjects. In contrast to cortical midline regions, other cortical regions showed differences in PBRs during EP and/or EJ. Smaller PBRs in depressed patients were observed in the occipital cortex bilaterally during both EP and EJ and in the left DLPFC during EJ. Larger signal increases in depressed patients were observed in the right
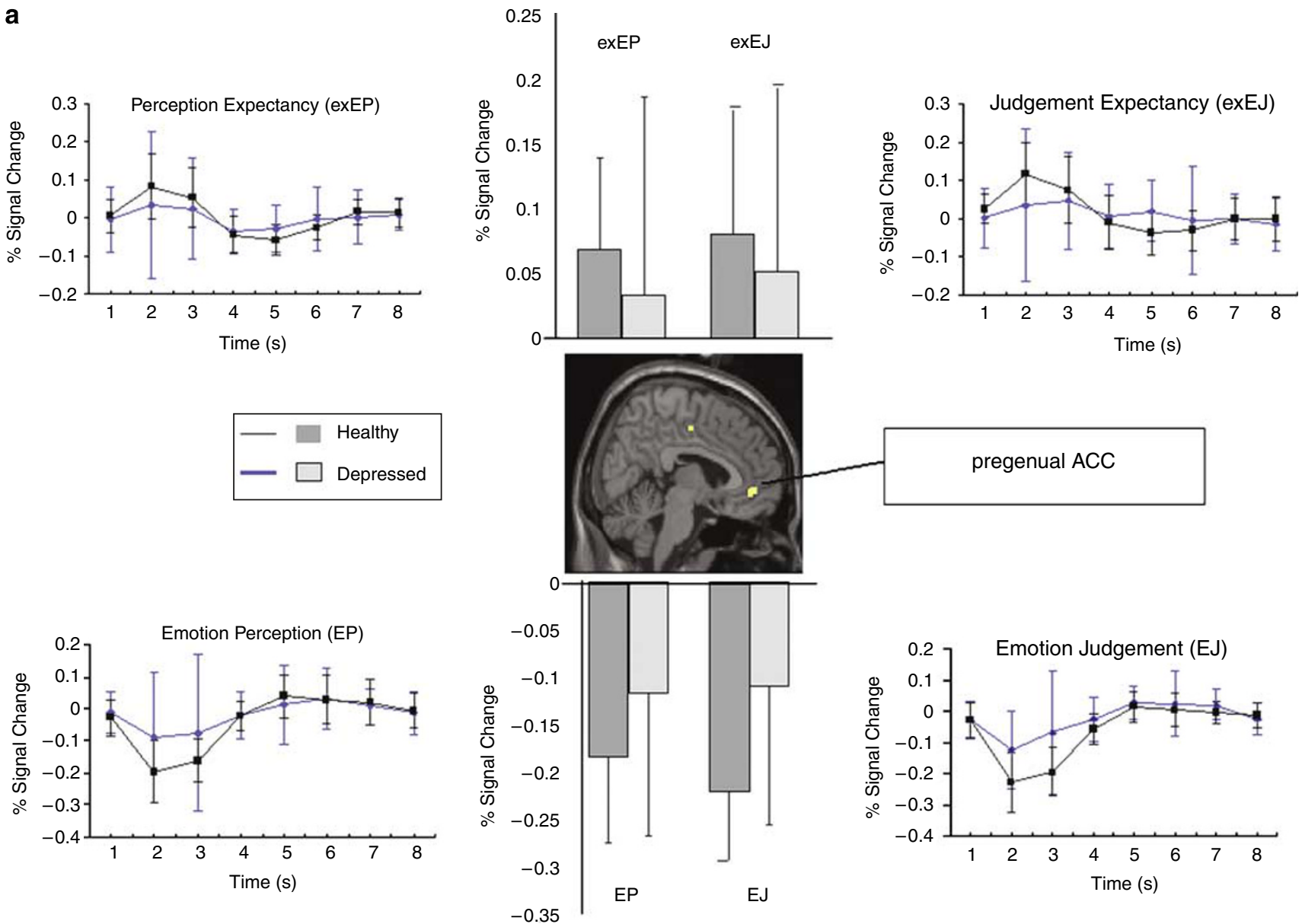

Figure 2 (a, b) Images show statistical parametric ( $T$ ) maps for the contrast rest $>$ all pictures (for healthy $>$ depressive subjects), overlaid on a single subject's normalized brain in the MNI stereotactic space. The global height threshold was set to $p<0.00$ I uncorrected with an extent threshold of $k=5$ voxels. The saggital images represent the right hemisphere. Bar diagrams (\% signal change representing means and SD) and corresponding time courses (\% signal change representing means and SD) are demonstrated for emotion perception (EP), emotion judgment (EJ), and the two expectancy conditions (ExEP and ExEJ) in the PACC $(10,46$, and -8$)$ and dorsal PCC $(8,-10$, and 46). 

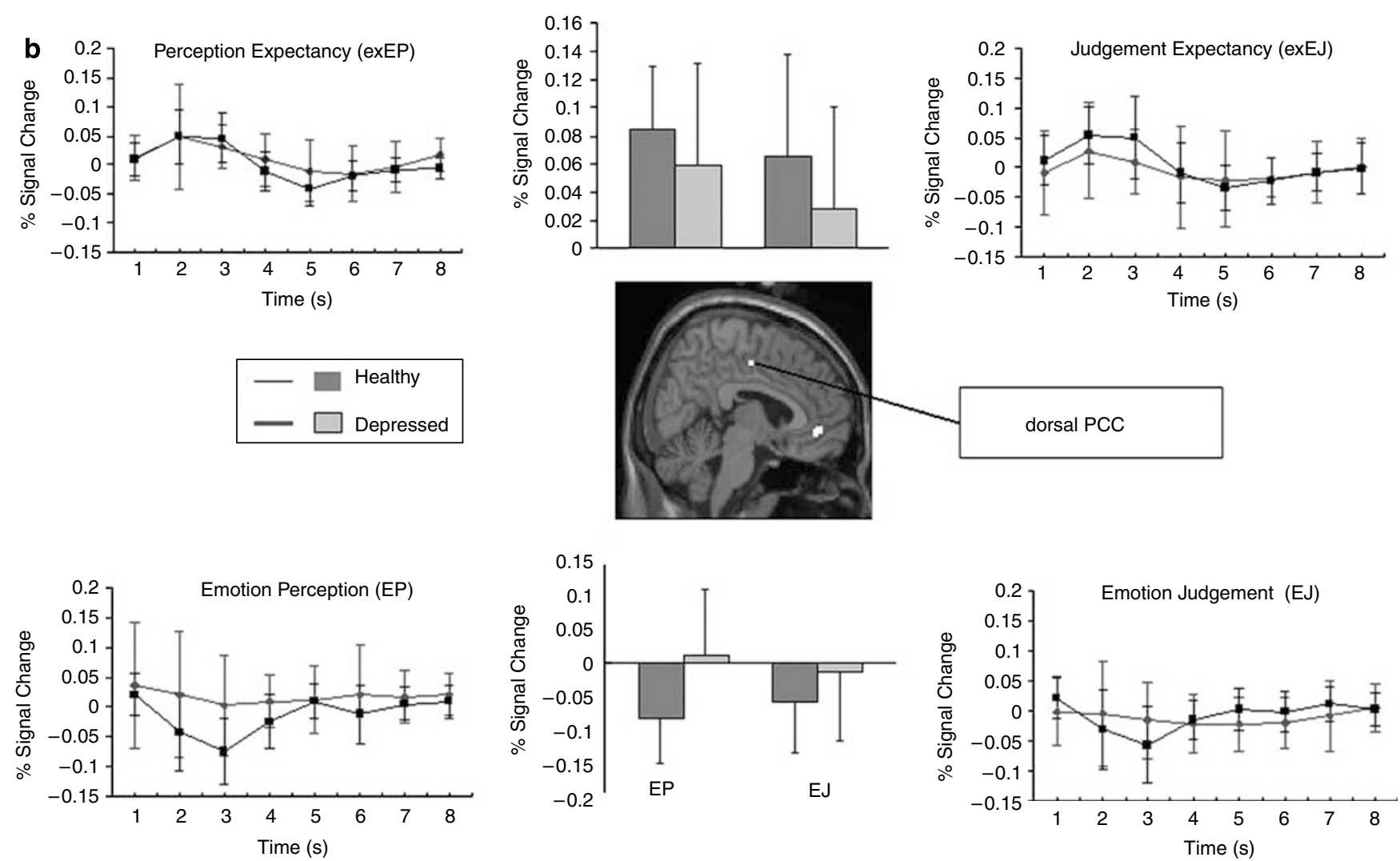

Figure 2 Continued.

DLPFC during EJ (Grimm et al, 2008). To confirm the finding of task-independent NBRs in CMS (Figure 2a and b), we performed a conjunction analysis between rest $>\mathrm{EJ}$ and rest $>\mathrm{EP}$ for both healthy and depressed subjects. In healthy subjects, the conjunction analysis revealed overlapping voxels at $p<0.05$ FWE-corrected in PACC/VMPFC $(6,46$, and -2$)$, dorsal PCC $(8,-14$, and 42$)$ as well as in bilateral superior/middle temporal gyrus $(42,-32$, and $6 ;-60,-28$, and -2$)$. Common activation in the PACC extending into the $\operatorname{VMPFC}(16,50$, and -4$)$ and medial parietal cortex $(6,-32$, and 66) was revealed in MDD subjects. To further underline these findings, the contrasts between rest $>\mathrm{EP}$ and rest $>$ EJ were exclusively masked with each other. Such masking analysis removes all voxels from the contrast of interest that reached a significance level of $p<0.05$ FWE-corrected in the masking contrast. The exclusive masking analysis of (rest $>E P$ ) with (rest $>E J)$ revealed a cluster of activation in the retrosplenial cortex $(-10,-52$, and 12) in healthy subjects and in medial temporal gyrus $(36,-28$, and -18$)$ and retrosplenial cortex $(-14,-58$, and $10)$ in depressed subjects. The opposite masking analysis of (rest $>$ EJ) with (rest $>$ EP) showed activation in the right parietal cortex $(56,-44$, and 28$)$ in healthy subjects and in superior temporal gyrus $(52,-50$, and 20) and precuneus $(0,-62$, and 48$)$ in depressed subjects. Results in the masking analysis of depressed subjects could only be obtained if the threshold was lowered to $p<0.001$ uncorrected. Regions of the default-mode network such as the PACC, VMPFC, and dorsal PCC are therefore involved in EP as well as in EJ, whereas more posterior CMS such as the retrosplenial cortex are specifically associated with EP. Negative BOLD responses in our ROIs, the CMS, were obtained during both EP and EJ. Because of the taskindependent signal changes during emotional stimulation, the two conditions were combined in the contrast rest $>$ all pictures and further analyses for the relationship of NBRs with psychopathological parameters and emotional valences were conducted using this contrast. Similarly to healthy subjects, depressed patients showed PBRs rather than NBRs in CMS during both expectancy periods (expectancy for EP and EJ; ExEP and ExEJ). During the expectancy periods, more PBRs in MDD subjects were observed in the VMPFC, whereas less PBRs were found in the dorsal anterior cingulate cortex and the left DLPFC (Table 1). Smaller PBRs in depressed patients when compared to healthy subjects were also found in the ROI analyses of the signal changes in PACC and dorsal PCC (as visible in the bar diagrams in Figure $2 a$ and $b$ ), even though differences did not reach statistical significance in SPM whole-brain analysis.

Even though not in the focus of our study, for the sake of completeness the direct comparison of EJ and EP (EJ > EP) between MDD and healthy subjects is to be mentioned. MDD patients showed significantly lower signal intensities in the left DLPFC $(-42,10$, and 30$)$, the left parietal cortex $(-38,-30$, and 50$)$, and the right occipital cortex $(18,-78$, and 24). Higher signal intensities in depressed subjects were found in the right DLPFC $(48,28$, and 0$)$, the right parietal cortex $(56,-56$, and 30$)$ and the precuneus $(0,-72$, and 38) (Grimm et al, 2008). 

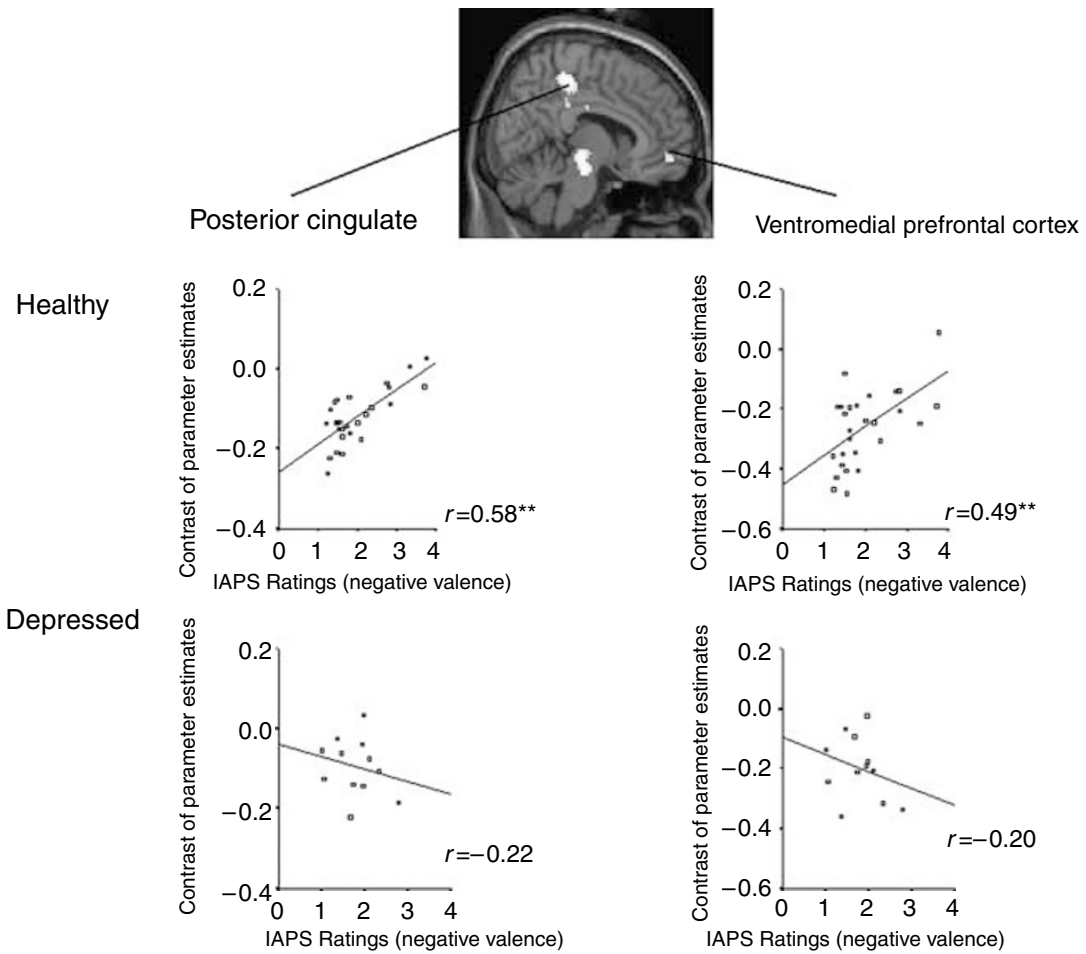

Figure 3 SPM image shows regions of positive correlation between healthy subjects' postscanning valence ratings for negative pictures and single-subject contrasts of parameter estimates for the contrast rest $>$ all pictures $(p<0.005$; uncorrected; $k>10)$. The saggital view represents the right hemisphere. Scatter plots show correlation of negative emotional valences with neural activity in the VMPFC (bordering on the PACC) $(8,52$, and -8$)$ and the PCC $(6,-32$, and 52$)$ for both healthy and depressed subjects. $* * * 0.001$

\section{Correlation of Signal Changes with Subjective Ratings of Emotional Valence}

Healthy subjects . To link the NBRs in the default-mode network to emotional valences, subjective postscanning ratings of emotional valence for negative and positive pictures were separately correlated with signal intensities in the contrast rest $>$ all pictures, which revealed a significant correlation for negative emotional valences in the VMPFC, bordering on the PACC and the PCC (Figure 3). Stronger NBRs in VMPFC $(r=0.58)$ and PCC $(r=0.49)$ were accompanied by more negative ratings of negative emotional pictures. Further regions of correlation included the right superior temporal gyrus $(r=0.59)$, retrosplenial cortex $(r=0.49)$, and subcortical regions (thalamus $(r=0.40)$ and tectum $(r=0.67)$, VTA extending to substantia nigra $(r=0.64)$ (Figure 4)). In contrast to negative emotional valences, we did not observe any correlation of signal changes in default-mode regions with positive emotional valences.

Subjects with $M D D$. In contrast to healthy subjects, MDD subjects showed no significant correlation of signal changes in VMPFC, PCC, VTA, and tectum with negative emotional valence in the contrast rest $>$ all pictures (Figures 3 and 4). In fact, the direction of correlation tended to be reversed with decreased NBRs being accompanied by more negative picture ratings. A comparison of correlation coefficients using Fischer's $z$-transformation confirmed significant differences in correlation between groups (PCC: $z=-2.79$, $p<0.001$; VMPFC: $z=-2.33, p<0.001$; VTA: $z=-4.63$, $p<0.001$; tectum: $z=-2.52, p<0.001)$.

\section{Correlation of NBRS with Psychopathological Parameters}

In a post hoc, ROI analysis, the correlation between duration of the current episode, number of previous episodes, patients' scores on the HDRS, BDI, BHS, and signal changes in PACC, VMPFC, and PCC was analyzed. MDD patients' subjective ratings of hopelessness (BHS score) correlated significantly with signal changes in the VMPFC $(r=-0.79$, $p=0.02$ ) and patients' scores on the Hamilton Depression Scale (HDRS score) with signal changes in the PCC $(r=-0.62, p=0.007)$ during rest $>$ all pictures: decreased NBRs were accompanied by stronger feelings of hopelessness and more severe depressive symptoms. Concerning the relationship of the different psychopathological components of the HDRS with NBRs, we found a significant correlation of the factor 'psychic depression', which includes items that reflect depressed mood, depressive cognitions, and suicidal tendency with signal changes in the PACC $(r=-0.53, p=0.03)$ during rest $>$ all pictures. There were no significant correlations for the other four factors that reflect different symptom clusters. Also, no correlations in the default-mode network regions could be found for the parameters of depression refractoriness (duration of the current episode and number of previous episodes) and BDI score. 


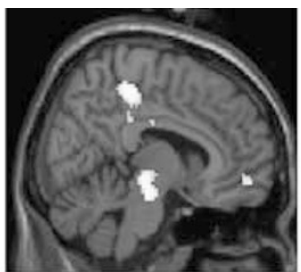

$\underline{\text { Tectum }}$

VTA

Healthy

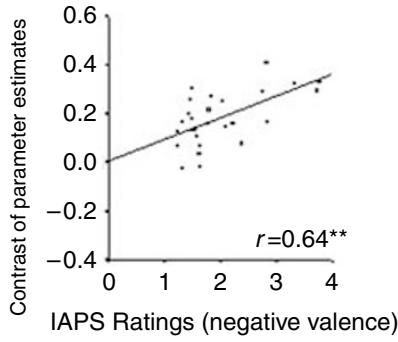

Depressed

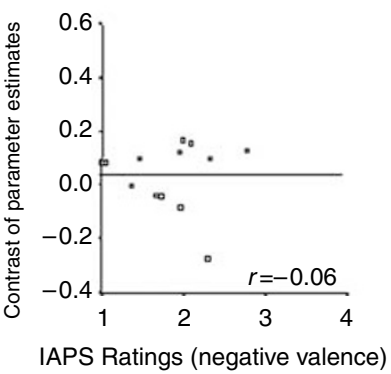

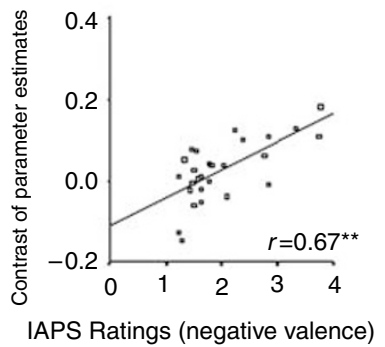

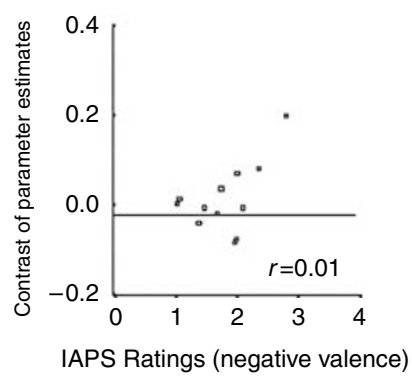

Figure 4 SPM image shows regions of positive correlation between healthy subjects' postscanning valence ratings for negative pictures and single-subject contrasts of parameter estimates for the contrast rest $>$ all pictures $(p<0.005$; uncorrected; $k>10)$. The saggital view represents the right hemisphere. Scatter plots show correlation of negative emotional valences with neural activity in the ventral tegmental area $(V T A)(8,-22$, and -6$)$ and the tectum $(-6,-20$, and -18$)$ for both healthy and depressed subjects. $* * * 0.001$.

\section{DISCUSSION}

This study investigated neural activity in the default-mode network during different emotional tasks in healthy subjects and patients with MDD in an event-related fMRI design. For both types of external emotional stimulation (EP and EJ), significant negative BOLD responses were found in the PACC, VMPFC, and dorsal PCC in both groups with NBRs significantly reduced in MDD patients. Decreased NBRs in the PCC and VMPFC in MDD patients correlated with depression severity and feelings of hopelessness. NBRs in the VMPFC correlated with ratings of negative emotional valence (but not with positive emotional valence) in healthy subjects, whereas this relationship was not found in MDD patients.

Previous studies demonstrated NBRs in default-mode network during various emotional as well as cognitive tasks (Simpson et al, 2001; Gusnard et al, 2001; McKiernan et al, 2003; Northoff et al, 2004; Raichle and Gusnard, 2005; Fransson, 2005; Grimm et al, 2006). In accordance with these observations, we found NBRs in PACC, VMPFC, and dorsal PCC during both EP and EJ thus indicating task independence. As hypothesized, MDD patients showed significantly decreased NBRs in the default-mode network during both EP and EJ when compared to healthy subjects. The origin of reduced NBRs remains unclear though. Resting-state studies using PET demonstrated increased metabolism in the VMPFC and the PAAC in MDD
(Mayberg, 2002, 2003; Phillips et al, 2003). The fact that we found decreased NBRs in VMPFC and PACC indicates that these could possibly be traced back to abnormally high resting-state neural activity rather than to a deficit in inducing NBR in response to emotional stimuli. This assumption is further supported by a recent fMRI study (Greicius et al, 2007) showing default-mode network abnormalities with increased resting-state subgenual cingulate functional connectivity in major depression. Abnormally high resting-state neural activity leading to decreased NBRs in PACC and VMPFC is also in keeping with studies showing relative hypermetabolism in these regions during emotional stimulation. MDD patients showed increased signal intensity in anterior cingulate cortex and ventral prefrontal cortex during the voluntary downregulation of sad feelings, the viewing of sad facial expressions, and the presentation of sad words (Canli et al, 2004; Lawrence et al, 2004; Surguladze et al, 2005, Beauregard et al, 2006). Also, previous studies that investigated neural activity as a function of antidepressant drug therapy report an association of treatment response with a decrease in fMRI activation or glucose metabolism in ventromedial and dorsomedial prefrontal cortex, and anterior cingulate (Robertson et al, 2007; Kennedy et al, 2007; Fu et al, 2004). Abnormal NBRs in MDD might therefore be state-related and normalize in remission. This assumption has to be tested by future studies, though. 
In contrast to EP and EJ, we did not observe NBRs but rather PBRs during emotional expectancy. This difference between conditions with (perception and judgment) and without (expectancy) an explicit emotional stimulus might psychologically be related to the switch between extrospectively and introspectively oriented modes of emotion processing. Emotional expectancy requires introspection of one's own mental states whereas, due to the presentation of emotional pictures, extrospection becomes predominant in $\mathrm{EP}$ and $\mathrm{EJ}$.

The finding of decreased NBRs in MDD suggests specific involvement of NBRs in processing negative emotions, which is substantiated by the results of the correlation analyses with subjects' ratings of emotional valence. Healthy subjects' NBRs in ventral CMS correlated with their ratings of negative emotional valence; stronger NBRs in the VMPFC were associated with more negative valence ratings. This relationship was found only for negative emotional valences, whereas we did not observe a correlation in positive emotional valences. This is in good agreement with previous studies that observed a correlation between signal changes in ventral CMS and negative emotional valences (Heinzel et al, 2005; Anderson et al, 2003; Lewis et al, 2006; Grimm et al, 2006). In contrast to healthy subjects, MDD subjects did not show a significant correlation between NBRs and negative valence ratings. This indicates that MDD subjects might thus no longer be able to appropriately modulate their negative emotions by NBRs in ventral CMS in a subtle and fine-grained way that allows them to distinguish between different degrees of emotional negativity. This assumption is supported by the significant correlation of depression severity (HDRS score) and patients' subjective ratings of hopelessness (BHS score) with signal changes in the VMPFC and PCC. For the PACC, there was a significant correlation of the signal changes with the symptom cluster 'psychic depression' (HDRS component; Milak et al, 2005), which comprises of symptoms such as depressed mood, depressive cognitions, and suicidal tendency. Decreased NBRs were accompanied by stronger feelings of hopelessness and more severe depressive symptoms. We therefore infer that weaker NBRs in CMS might be specifically involved in processing abnormally negative emotions in MDD.

One limitation in our study is that we cannot fully exclude that weaker NBRs in MDD subjects could also result from reduced motivation or attention during task performance. Even though we obtained slower reaction times in MDD subjects, these did not correlate with signal intensities in CMS. Also, the variance in CMS signal intensities attributable to reaction times was covaried out in an ANOVA test. MDD patients' performance of the expectancy task (as mirrored in the induction of PBRs during the expectancy period) also argue against general motivational deficits.

Because of the fact that the judgment task was biased by requiring the subject to respond with the left hand for pictures judged as negative and the right hand when the picture was judged positive, it is possible that there was an affective congruence effect leading to more right hemisphere arousal during negative stimuli and more left hemisphere arousal during positive stimuli. In line with the valence-lateralization theory, which postulates a dominance of the left prefrontal cortex in positive emotions and of the right prefrontal cortex in negative emotions (Davidson et al, 2003; Murphy et al, 2003; Wager et al, 2003), fMRI studies in healthy subjects demonstrated linear or parametric dependence of negative and positive EJs on neural activity in left and right DLPFC (Grimm et al, 2006). The hemispheric mood congruence effect is further supported by symmetrical responses during the perception task, where subjects were asked to respond with either hand. Interestingly though, in MDD patients the effect is more pronounced in positive stimuli, whereas healthy subjects show the opposite pattern with more symmetrical responses for negative stimuli.

Another limitation of this study pertains to the discordant exposure to medications between healthy controls and depressed patients. It is conceivable that differences in NBRs between these two groups reflect the fact that patients had been taking psychotropic medications until a mean of 9 days before the fMRI investigation. The length of this period might not have been sufficient to wash out psychotropic medication effects. Arguing against this potential confound is the fact that NBRs in patients who were antidepressant naïve $(N=3)$ or had a wash-out period of 2 weeks or more $(N=10)$ did not differ significantly. Nonetheless, we cannot completely refute the potential confound of medication exposure in the current study. The ideal study, which should contrast controls with either antidepressantnaive patients or those, who had a wash-out period of at least 2 weeks (8 weeks for patients on fluoxetine) would face substantial practical and ethical hurdles though.

In sum, we demonstrate reduced NBRs in core cortical midline regions of the default-mode network in MDD. As reduced NBRs also correlated with subjective measures of emotional valence and depressive symptoms, our results lend evidence to the assumption that reduced cortical midline NBRs are crucially implicated in abnormal negative emotional processing in MDD.

\section{ACKNOWLEDGEMENTS}

The study was supported by a Heisenberg grant from the German Research Foundation (DFG, 304/4-1 to GN), the Swiss National Research Foundation (3100A0-100830) to GN and HB, a grant of the Research Foundation at the University of Zurich, Organon, Switzerland, to GN and HB, a grant of the Salus Foundation Sachsen Anhalt/Germany to GN, a grant from ETH Zurich (SEP) and Philips Medical Systems, Best, NL, to PB, the Hartmann-Müller-Foundation to $\mathrm{HB} / \mathrm{GN}$, the Gebert-Rüf-Foundation to $\mathrm{HB} / \mathrm{GN}$, and the German Federal Ministry of Education and Research (BMBF-01GWSO61 to FB) as well as a grant from the Hope of Depression Research Foundation (HDRF/ISAN) to GN.

\section{DISCLOSURE/CONFLICT OF INTEREST}

The authors report no conflict of interests.

\section{REFERENCES}

Anderson AK, Christoff K, Stappen I, Panitz D, Ghahremani DG, Glover G et al (2003). Dissociated neural representations of intensity and valence in human olfaction. Nat Neurosci 6: 196-202. 
Beauregard M, Paquette V, Levesque J (2006). Dysfunction in the neural circuitry of emotional self-regulation in major depressive disorder. NeuroReport 17: 843-846.

Bechara A, Tranel D, Damasio H, Adolphs R, Rockland C, Damasio AR (1995). Double dissociation of conditioning and declarative knowledge relative to the amygdala and hippocampus in humans. Science 269: 1115-1118.

Beck AT, Ward CH, Mendelson M, Mock J, Erbaugh J (1961). An inventory for measuring depression. Arch Gen Psychiatry 4: 561-571.

Beck AT, Weissman A, Lester D, Trexler L (1974). The measurement of pessimism: the hopelessness scale. J Consult Clin Psychol 42: 861-865.

Bermpohl F, Fregni F, Boggio PS, Thut G, Northoff G, Otachi PTM et al (2006). Effect of low-frequency transcranial magnetic stimulation on an affective go/no-go task in patients with major depression: role of stimulation site and depression severity. Psychiatry Res 141: 1-13.

Canli T, Sivers H, Thomason ME, Whitfield-Gabrieli S, Gabrieli JD, Gotlib IH (2004). Brain activation to emotional words in depressed vs healthy subjects. NeuroReport 15: 2585-2588.

Davidson RJ, Irwin W, Anderle MJ, Kalin NH (2003). The neural substrates of affective processing in depressed patients treated with venlafaxine. Am J Psychiatry 160: 64-75.

Dunn RT, Kimbrell TA, Ketter TA, Frye MA, Willis MW, Luckenbaugh DA et al (2002). Principal components of the Beck depression inventory and regional cerebral metabolism in unipolar and bipolar depression. Biol Psychiatry 51: 387-399.

Fox MD, Snyder AZ, Vincent JL, Corbetta M, Van Essen DC, Raichle ME (2005). From the cover: the human brain is intrinsically organized into dynamic, anticorrelated functional networks. Proc Natl Acad Sci USA 102: 9673-9678.

Fox MD, Snyder AZ, Zacks JM, Raichle ME (2006). Coherent spontaneous activity accounts for trial-to-trial variability in human evoked brain responses. Nat Neurosci 9: 23-25.

Fransson P (2005). Spontaneous low-frequency BOLD signal fluctuations: an fMRI investigation of the resting-state default mode of brain function hypothesis. Hum Brain Mapp 26: 5-29.

Fu CHY, Williams SCR, Cleare AJ, Brammer MJ, Walsh ND, Kim J et al (2004). Attenuation of the neural response to sad faces in major depression by antidepressant treatment: a prospective, event-related functional magnetic resonance imaging study. Arch Gen Psychiatry 61: 877-889.

Greicius MD, Flores BH, Menon V, Glover GH, Solvason HB, Kenna $\mathrm{H}$ et al (2007). Resting-state functional connectivity in major depression: abnormally increased contributions from subgenual cingulate cortex and thalamus. Biol Psychiatry 62: 429-437.

Greicius MD, Srivastava G, Reiss AL, Menon V (2004). Defaultmode network activity distinguishes Alzheimer's disease from healthy aging: evidence from functional MRI. Proc Natl Acad Sci USA 101: 4637-4642.

Grimm S, Beck J, Schuepbach D, Hell D, Boesiger P, Bermpohl F et al (2008). Imbalance between left and right dorsolateral prefrontal cortex in major depression is linked to negative emotional judgment: an fMRI study in severe major depressive disorder. Biol Psychiatry 63: 369-376.

Grimm S, Schmidt CF, Bermpohl F, Heinzel A, Dahlem Y, Wyss M et al (2006). Segregated neural representation of distinct emotion dimensions in the prefrontal cortex - an fMRI study. NeuroImage 30: 325-340.

Gusnard DA, Raichle ME, Raichle ME (2001). Searching for a baseline: functional imaging and the resting human brain. Nat Rev Neurosci 2: 685-694.

Hamilton M (1960). A rating scale for depression. J Neurol Neurosurg Psychiatry 23: 56-62.

Heinzel A, Bermpohl F, Niese R, Pfennig A, Pascual-Leone A, Schlaug $\mathrm{G}$ et al (2005). How do we modulate our emotions?
Parametric fMRI reveals cortical midline structures as regions specifically involved in the processing of emotional valences. Cogn Brain Res 25: 348-358.

Kennedy SH, Evans KR, Kruger S, Mayberg HS, Meyer JH, McCann S et al (2001). Changes in regional brain glucose metabolism measured with positron emission tomography after paroxetine treatment of major depression. Am J Psychiatry 158: 899-905.

Kennedy SH, Konarski JZ, Segal ZV, Lau MA, Bieling PJ, McIntyre RS et al (2007). Differences in brain glucose metabolism between responders to CBT and venlafaxine in a 16-week randomized controlled trial. Am J Psychiatry 164: 778-788.

Kimbrell TA, Ketter TA, George MS, Little JT, Benson BE, Willis MW et al (2002). Regional cerebral glucose utilization in patients with a range of severities of unipolar depression. Biol Psychiatry 51: 237-252.

Lang PJ, Bradley MM, Cuthbert BN (1999). International Affective Picture System (IAPS) [Database].

Lawrence NS, Williams AM, Surguladze S, Giampietro V, Brammer MJ, Andrew C et al (2004). Subcortical and ventral prefrontal cortical neural responses to facial expressions distinguish patients with bipolar disorder and major depression. Biol Psychiatry 55: 578-587.

Lewis PA, Critchley HD, Rotshtein P, Dolan RJ (2006). Neural correlates of processing valence and arousal in affective words. Cereb Cortex 17(3); 742-748.

Logan BR, Rowe DB (2004). An evaluation of thresholding techniques in fMRI analysis. Neurolmage 22: 95-108.

Mayberg H (2002). Depression, II: localization of pathophysiology. Am J Psychiatry 159: 1979.

Mayberg HS (2003). Modulating dysfunctional limbic-cortical circuits in depression: towards development of brain-based algorithms for diagnosis and optimised treatment. Br Med Bull 65: 193-207.

McKiernan KA, Kaufman JN, Kucera-Thompson J, Binder JR (2003). A parametric manipulation of factors affecting taskinduced deactivation in functional neuroimaging. $J \operatorname{Cog} n$ Neurosci 15: 394-408.

Milak MS, Parsey RV, Keilp J, Oquendo MA, Malone KM, Mann JJ (2005). Neuroanatomic correlates of psychopathologic components of major depressive disorder. Arch Gen Psychiatry 62: 397-408.

Moran JM, Macrae CN, Heatherton TF, Wyland CL, Kelley WM (2006). Neuroanatomical evidence for distinct cognitive and affective components of self. J Cogn Neurosci 18: 1586-1594.

Murphy FC, Nimmo-Smith I and Lawrence AD (2003). Functional neuroanatomy of emotions: a meta-analysis. Cogn Affect Behav Neurosci 3: 207-233.

Northoff G, Heinzel A, Bermpohl F, Niese R, Pfennig A, PascualLeone A et al (2004). Reciprocal modulation and attenuation in the prefrontal cortex: an fMRI study on emotional-cognitive interaction. Hum Brain Mapp 21: 202-212.

Oldfield RC (1971). The assessment and analysis of handedness: the Edinburgh inventory. Neuropsychologia 9: 97-113.

Ollinger JM, Shulman GL, Corbetta M (2001). Separating processes within a trial in event-related functional MRI. NeuroImage 13: 210-217.

Phan KL, Taylor SF, Welsh RC, Ho SH, Britton JC, Liberzon I (2004). Neural correlates of individual ratings of emotional salience: a trial-related fMRI study. NeuroImage 21: 768-780.

Phillips ML, Drevets WC, Rauch SL, Lane R (2003). Neurobiology of emotion perception II: implications for major psychiatric disorders. Biol Psychiatry 54: 515-528.

Price CJ, Friston KJ (1997). Cognitive conjunction: a new approach to brain activation experiments. NeuroImage 5: 261-270.

Pruessmann KP, Weiger M, Scheidegger MB, Boesiger P (1999). SENSE: sensitivity encoding for fast MRI. Magn Reson Med 42: 952-962. 
Raes F, Hermans D, Williams JM, Demyttenaere K, Sabbe B, Pieters G et al (2006). Is overgeneral autobiographical memory an isolated memory phenomenon in major depression? Memory 14: 584-594.

Raichle ME, Gusnard DA (2005). Intrinsic brain activity sets the stage for expression of motivated behavior. J Comp Neurol 493: 167-176.

Raichle ME, MacLeod AM, Snyder AZ, Powers WJ, Gusnard DA, Shulman GL (2001). A default mode of brain function. Proc Natl Acad Sci USA 98: 676-682.

Rajkowska G (2002). Cell pathology in mood disorders. Semin Clin Neuropsychiatry 7: 281-292.

Rajkowska G (2003). Depression: what we can learn from postmortem studies. Neuroscientist 9: 273-284.

Rimes KA, Watkins E (2005). The effects of self-focused rumination on global negative self-judgements in depression. Behav Res Ther 43: 1673-1681.

Robertson B, Wang L, Diaz MT, Aiello M, Gersing K, Beyer J et al (2007). Effect of bupropion extended release on negative emotion processing in major depressive disorder: a pilot functional magnetic resonance imaging study. J Clin Psychiatry 68: 261-267.
Schmidt CF, Boesiger P and Ishai A (2005). Comparison of fMRI activation as measured with gradient- and spin-echo EPI during visual perception. Neuroimage 26: 852-859.

Simpson SW, Baldwin RC, Burns A, Jackson A (2001). Regional cerebral volume measurements in late-life depression: relationship to clinical correlates, neuropsychological impairment and response to treatment. Int J Geriatr Psychiatry 16: 469-476.

Stark CE, Squire LR (2001). When zero is not zero: the problem of ambiguous baseline conditions in fMRI. Proc Natl Acad Sci USA 98: $12760-12766$

Surguladze SA, Young AW, Senior C, Brebion G, Travis MJ, Phillips ML (2005). Recognition accuracy and response bias to happy and sad facial expressions in patients with major depression. Neuropsychology 18: 212-218.

Wager TD, Phan KL, Liberzon I, Taylor SF (2003). Valence, gender, and lateralization of functional brain anatomy in emotion: a meta-analysis of findings from neuroimaging. Neuroimaage 19: 513-531.

Treynor W (2003). Rumination reconsidered: a psychometric analysis. Cogn Ther Res 27: 247-259. 